\title{
Reciprocal effects of visual and auditory stimuli in a spatial compatibility situation
}

\author{
RICHARD RAGOT \\ Hôpital de la Salpêtrière, Paris, France \\ CHRISTIAN CAVE \\ Université de Marseille 1, Aix-en-Provence, France \\ and \\ MICHEL FANO \\ Commission Supérieure Technique du Cinema, Paris, France \\ (Charles W. Eriksen, Sponsor)
}

\begin{abstract}
When events occur in the visual and auditory modalities simultaneously, normal humans often give preference to the visually presented information. In the present experiment the existence of such a visual predominance was tested in a spatial compatibility paradigm. The results showed that reaction times were significantly delayed when the position of the visual stimulus did not coincide with the position of the auditory stimulus, whether subjects attended to the visual or to the auditory stimuli. Thus, no visual predominance was demonstrated. The discrepancy between these results and those of studies showing visual predominance can be explained by differences in the direction of attention: visual predominance appears when attention is divided between visual and auditory modalities, but seems to be absent (as in the present experiment) when subjects are asked to attend to one modality while ignoring the other.
\end{abstract}

The prepotency of the visual over the auditory sensory system has been observed in many experimental situations in normal humans. One such situation produces what can be referred to as the "ventriloquist effect": a sound is perceived as if it were coming from a point in the visual environment where something is moving or changing, even when the physical source of the sound is elsewhere. Such an effect takes place, for example, in a television receiver, where speech production is naturally attributed to the characters appearing on the screen despite the fact that the loudspeaker is located to the side of and not right behind the screen. This effect persists over a fairly wide range of distances between the acoustic and visual sources (Jackson, 1953), although it can disappear if gross incongruities between them are introduced (e.g., by placing the loudspeaker behind the spectator).

Experiments by Colavita $(1974,1982)$ showed visual predominance to be a strong effect. Replicating Colavita's results with variations in the experimental conditions, Posner, Nissen, and Klein (1976) and Egeth and Sager (1977)

This research was supported by Grant 955540 (ATP) from the Centre National de la Recherche Scientifique. The authors wish to thank Nicole Lesèvre and Mark Smith for their critical reading and suggestions concerning the manuscript. Request reprints from Richard Ragot, Laboratoire d'Electrophysiologie et de Neurophysiologie Appliquée, CNRS UA 654 LENA, Hôpital de la Salpêtrière, 47, bd de l'Hôpital, 75651 Paris, Cedex 13, France. suggested that visual predominance may be caused by a general attentional bias toward the visual modality that would compensate for the apparently low alerting capability of visual signals.

The study of stimulus-response (S-R) compatibility provides other experimental paradigms that can be used to investigate the relative potency of two sensory systems or subsystems. Spatial S-R incompatibility effects can occur in choice reaction time (RT) situations when a subject is required to make a response in a direction different from that of the incoming stimulus. In this case, a lengthening of both the RT (Simon, 1969) and the latency of the P300 component of the visual evoked brain potential is obtained (Ragot, 1984; Ragot \& Lesèvre, 1986). In the case of a lateralized visual stimulus, Simon and Craft (1970) and Simon (1982) found that the presentation of a sound can have a delaying effect on the RT if the sound is presented to the ear opposite to the visual stimulus, but they did not specifically test the inverse condition, that is, the presentation of distracting visual stimuli during an auditory task.

Since visual predominance has been shown by Colavita (1974, 1982), Colavita, Tomko, and Weisberg (1976), Posner et al. (1976), and Egeth and Sager (1977) to be a consistent and robust phenomenon, it would be of interest to know whether visual predominance would persist in situations of spatial incompatibility: How else could one explain that vision can be affected by simulta- 
neous auditory information in a paradigm such as Simon and Craft's (1970)? The purpose of the present experiment was to extend Simon and Craft's study by (1) investigating the reciprocal effects of sound on light, and vice versa, in a spatial compatibility paradigm, and (2) testing whether visual predominance is an attentionmodulated phenomenon.

Predominance was studied by presenting the subjects with trials in which sound and light location did not coincide, whereas attentional bias was manipulated by asking the subjects to respond either only to light or only to sound (in two distinct blocks), using visual and auditory stimuli of the same intensity in the two blocks.

\section{METHOD}

Four male and 4 female right-handed volunteers, ranging in age from 20 to 42, served as subjects for the experiment. All subjects reported having normal hearing, normal color vision, and either normal or corrected-to-normal visual acuity. The subjects were seated in a soundattenuating room, facing a gray display panel placed vertically at a distance of $126 \mathrm{~cm}$ in front of their eyes, and were asked to keep looking at a central red light-emitting diode (LED) situated in the middle of the panel (the fixation point).

The absolute threshold of auditory perception is known to vary greatly with fatigue and among individuals (Dadson \& King, 1952), and the absolute threshold of visual perception also shows great variations with time and adaptation to darkness. Differential intensities in both modalities are known to be much smaller, and also much more stable (Stevens, 1957). Therefore, both visual and auditory stimuli were presented against a background sensory reference. This procedure allowed stimulus intensity to be expressed in relation to the background, rather than as absolute levels above threshold, and the same stimulus intensity value and stimulus-to-background-intensity ratio were used for all the subjects.

The sensory backgrounds consisted of the above-mentioned gray display panel for the visual stimulus and white noise for the auditory stimulus. The background illumination of the panel came from two $40-\mathrm{W}$ bulbs that provided a near-uniform luminance of $18 \pm 2 \mathrm{~cd} / \mathrm{m}^{2}$. The visual stimuli consisted of two red LED light bars $\left(.36^{\circ}\right.$ in diameter) located on the panel at a $20^{\circ}$ angle to the right and to the left of the fixation point. Stimulus intensity was $144 \mathrm{~cd} / \mathrm{m}^{2}$ (eight times that of the background), and stimulus duration was very short $(2 \mathrm{msec})$. A central loudspeaker, placed behind the visual fixation point, produced a continuous $60 \mathrm{~dB}$ SPL white noise. This served both as an auditory "fixation point" and as a background sound source against which the auditory stimuli were heard. The auditory stimuli consisted of very short ( $2 \mathrm{msec}$ duration) bursts of a 2-kHz sine wave tone (beep), which fed either of two loudspeakers located right and left (just behind the LEDs delivering the visual stimuli). Sound intensity was $78 \mathrm{~dB}$ SPL, that is, eight times louder than that of the auditory background.

Since each stimulation consisted of the simultaneous presentation of both a right or left visual signal and a right or left auditory beep, four possible combinations were produced. When visual and auditory stimuli occurred on the same side, the condition was said to be spatially compatible, whereas when the stimuli occurred on opposite sides, the condition was termed spatially noncompatible or conflictual.

Subjects had the index fingers of both hands resting on buttons (microswitches) placed $42 \mathrm{~cm}$ apart in front of them. In the auditoryattention situation, they were asked to pay attention only to the auditory component of the stimulus while trying to ignore its visual component, and to respond by pressing, as fast as possible, the button located on the same side as the auditory stimulus. In the visual-attention situation, subjects had to respond on the same side as the visual component of the stimulus, while trying to ignore the sound.

Stimuli were delivered at a constant rate of $2 \mathrm{sec}$, in five successive runs of 64 stimuli each. They were stored in computer memory in a pseudorandom order, and were arranged in such a way that there was the same number (16) of each category in each run. Subjects were allowed to rest between runs. The same set of $5 \times 64$ stimuli was used in both the visual and auditory situations. The situation order was reversed for half the subjects.

RTs were measured with a Venner TSA 6635 digital counter. Horizontal and vertical electrooculograms were recorded during the experiment, together with stimulus and response markers. This made it possible to discard errors and trials contaminated by eye movements.

\section{RESULTS}

A three-way repeated measures analysis of variance (ANOVA) was performed on the mean RT values of the 8 subjects. The factors were auditory/visual attention, compatible/incompatible conditions, and left/right responding hand. In the auditory-attention situation, RTs to compatible stimuli (i.e., sound and light appearing in the same place) were significantly shorter $(318 \mathrm{msec}$, pooled data for both hands) than those to incompatible stimuli (373 msec; Figure 1). In the visual-attention situation, RTs to compatible stimuli $(289 \mathrm{msec})$ were also shorter than RTs to incompatible stimuli $(347 \mathrm{msec})$. There was a main effect of RTs in response to compatible/ incompatible stimuli as tested globally on the 8 subjects with the ANOVA $[F(1,7)=76.71, p<.001]$, but no effect of either modality of attention $[F(1,7)=1.59]$ or responding hand $[F(1,7)=0.80]$. The only interaction observed was that of compatibility $\times$ responding hand $[F(1,7)=11.58, p<.0025]$.

Because of the large number of trials (80) performed in each experimental condition, a Student's $t$ test (with Aspin-Welch's correction for comparing data with different standard deviations) was used to assess individual subjects' responses. For each individual subject, RTs to compatible stimuli were also significantly shorter in both the auditory- and the visual-attention situations (all ps $<.001)$. Although some subjects were faster in the visual-attention than in the auditory-attention situation, the reverse was true for other subjects, so no significant tendency to be faster for one modality was observed

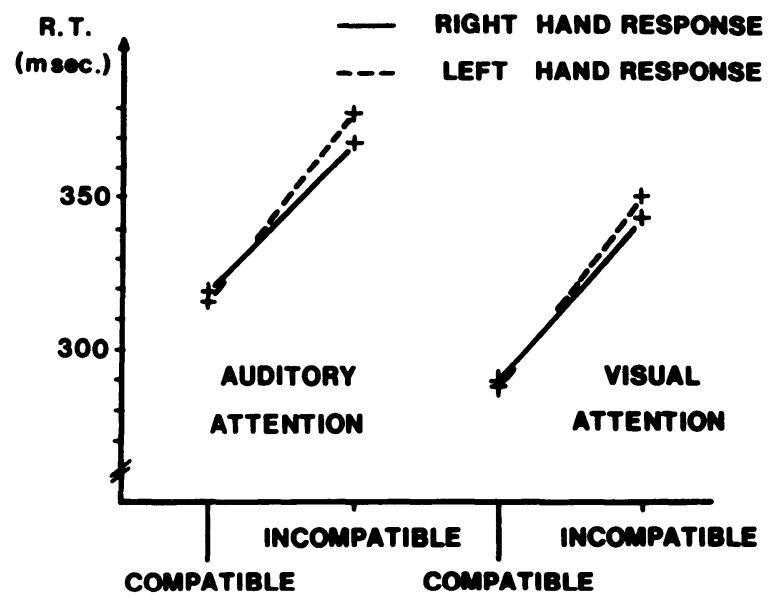

Figure 1. Average reaction time for the 8 subjects. 
among the 8 subjects tested $[F(1,7)=1.59)$. Errors in responding were minimal (on average, $2 \%$ of total responses), as were eye-movement-contaminated trials $(.4 \%)$.

\section{DISCUSSION}

The main result of the present study was that all of the subjects tested showed a significant RT increase when the spatial location of the two modalities did not coincide. The data concerning the delay induced in the visual-attention situation by noncompatible auditory stimuli are in accordance with the data of Simon and Craft (1970) and Simon (1982). An equivalent delay was caused by incompatible visual stimuli in the auditory-attention situation. There was therefore not only a disturbing effect of light in response to sound when the two stimuli did not coincide, but also a similar effect of sound in response to light. This reciprocal effect of one modality upon the other appears very robust, inasmuch as it was significant for each individual subject. Thus, in the present study, no predominance of one modality over the other was observed, contrary to the strong visual predominance observed by Colavita (1974, 1982)

Could the intensity ratio of light to sound explain the absence of visual predominance observed in our experiment, the visual stimulus being perceived as of lower intensity than the auditory stimulus? This explanation is not satisfactory for at least three reasons: (1) In order to try to equalize as much as possible the visual and auditory stimuli, their intensity was adjusted to be exactly the same amount ( 8 times) greater than that of their respective backgrounds. (2) It has been shown by Colavita (1974) and by Egeth and Sager (1977) that visual predominance was not greatly affected by manipulations of stimulus intensity. (3) Despite the fact that the ratio of light to sound intensity used in their experiments $\left(27.4 \mathrm{~cd} / \mathrm{m}^{2}\right.$ over $\left.65 \mathrm{~dB}\right)$ was about the same as ours, Egeth and Sager (1977) observed visual predominance.

Thus, the observation of visual predominance could not have been due to variations in stimulus intensity. It is more likely to have been caused, as we shall see, by differences in the experimental paradigms employed.

An explanation of visual dominance in terms of attentional mechanisms has been offered by Posner et al. (1976). Accordingly, we suggest that the discrepancy between Colavita's (1974) results and ours may be due to different attentional conditions. In our experiment, subjects had to keep their attention focused on one given modality during a whole block, and ignore all information from the other modality. In Colavita's experiment, on the contrary, attention was divided between the auditory and visual modalities, since subjects ignored whether the next stimulus would be auditory or visual (or both). We propose, therefore, that the visual predominance observed in Colavita's experiment could be attributed to differences between switching times for the two modalities. In Colavita's experiment, it is likely that it took less time to switch from divided to visual attention than from divided to auditory attention, since it was observed that bimodal stimuli were almost always identified as visual, which would indicate that visual information is available sooner than auditory information. In our experiment, no switching was necessary, because attention had to stay focused on one given modality for the duration of the block. If visual predominance is indeed due to differences in modality switching time, as we suggested above, it is logical that such a predominance was not observed in our experiment.

The significant interaction between compatibility and responding hand shown in the ANOVA remains to be interpreted. Indeed, in both the auditory- and visual-attention situations, the RT increase with incompatibility was greater when the response was given with the left hand (Figure 1). Because the left-hand muscles have more direct connections with the right hemisphere than with the left one, one possible explanation is that right-hemisphere-controlled responses may be more reactive to spatial conflict situations than those controlled by the left hemisphere. Such an interpretation is in agreement with numerous experiments demonstrating a greater involvement of the right hemisphere in spatial tasks, at least in right-handers (Geffen, Bradshaw, \& Wallace, 1971; Kimura, 1963; Milner, 1965; Pohl, Butters, \& Goodglass, 1972).

\section{REFERENCES}

Colavita, F. B. (1974). Human sensory dominance. Perception \& Psychophysics, 16, 409-412.

Colavita, F. B. (1982). Visual dominance and attention in space. Bulletin of the Psychonomic Society, 19, 261-262.

Colavita, F. B., Tomko, R., \& Weisberg, D. (1976). Visual prepotency and attention in space. Bulletin of the Psychonomic Society, 8, 25-26.

Dadson, R. S., \& KING, J. H. (1952). A determination of the normal threshold of hearing and its relation to the standardisation of audiometers. Journal of Laryngology \& Otology, 66, 366-378.

EGETH, H. E., \& SAGER, L. C. (1977). On the locus of visual dominance. Perception \& Psychophysics, 22, 77-86.

Geffen, G., Bradshaw, J. L., \& Wallace, G. (1971). Interhemispheric effects of reaction time to verbal and nonverbal visual stimuli. Journal of Experimental Psychology, 87, 415-422.

JACKSON, C. V. (1953). Visual factors in auditory localization. Quarterly Journal of Experimental Psychology, 64, 54-67.

KImURA, D. (1963). Right temporal-lobe damage. Archives of Neurology, 8, 264-271.

MILNER, B. (1965). Visually-guided maze learning in man: Effects of bilateral hippocampal, bilateral frontal, and unilateral cerebral lesions. Neuropsychologia, 3, 317-338.

PoHL, W., Butters, N., \& Goodglass, H. (1972). Spatial discrimination systems and cerebral lateralization. Cortex, 8, 305-314.

Posner, M. I., Nissen, M. J., \& Klein, R. M. (1976). Visual dominance: An information-processing account of its origins and significance. Psychological Review, 83, 157-171.

RAGOT, R. (1984). Perceptual and motor space representation: An eventrelated potential study. Psychophysiology, 21, 159-170.

RAGOT, R., \& LESÈvRE, N. (1986). Electrophysiological study of intrahemispheric compatibility effects elicited by visual directional cues. Psychophysiology, 23, 19-27.

Simon, J. R. (1969). Reactions toward the source of stimulation. Journal of Experimental Psychology, 81, 174-176.

Simon, J. R. (1982). Effect of an auditory stimulus on the processing of a visual stimulus under single- and dual-tasks conditions. Acta Psychologica, 51, 61-73.

Simon, J. R., \& CRAFT, J. L. (1970). Effects of an irrelevant auditory stimulus on visual choice reaction time. Journal of Experimental Psychology, 86, 272-274.

Stevens, S. S. (1957). On the psychophysical laws. Psychological Review, 64, 153-181.

(Manuscript received for publication November 2, 1987.) 